\title{
Evaluation of Comprehensive Medication Review Completion Rates Using 3 Patient Outreach Models
}

\author{
Daryl E. Miller, PharmD; Teresa E. Roane, PharmD, BCACP; \\ Jennifer A. Salo, PharmD, BCACP; and Heather C. Hardin, PharmD, BCACP
}

\begin{abstract}
BACKGROUND: Medication therapy management (MTM) programs were first introduced as a result of the 2003 Medicare Prescription Drug Improvement and Modernization Act. Since then, the Centers for Medicare \& Medicaid Services (CMS) have established minimum requirements for health plans to follow in establishing patient eligibility for enrollment in these programs. The eligibility criteria are based on projected annual Part $D$ medication costs, number of chronic disease states, and number of chronic Part D covered medications. Patients meeting these criteria are automatically enrolled in an MTM program so must ask to be disenrolled from the program if they wish to not participate (opt-out). CMS rates the quality of health plans using the Five-Star Quality Rating System. Star ratings for various metrics are given to health plans based on a scale of 1 to 5 stars, with 5 stars being the highest quality rating that health plans can achieve. The "Medication Therapy Management Program Completion Rate for Comprehensive Medication Reviews (Part D)" was a display measure and is now a performance measure as of 2016. Consumers can view the star ratings and display measures for their health plans during the enrollment period, so these ratings can affect patient enrollments. Additionally, star ratings may also determine bonus payments awarded to health plans. To address the comprehensive medication review (CMR) measure, different outreach models are used by health plans and vendors to complete CMRs. Comparison of the effectiveness of these outreach models has not been sufficiently studied.
\end{abstract}

OBJECTIVE: To compare the completion rate of CMRs by the University of Florida Medication Therapy Management Communication and Care Center (MTMCCC) for 3 models of patient outreach (cold calling, the initial offer and acceptance of a CMR from the health plan before contact by the MTMCCC, and scheduling of a CMR after mailing a welcome letter from the health plan) used for 3 different health plans.

METHODS: This study is a retrospective chart analysis using data obtained from completed CMRs at the MTMCCC for health insurance beneficiaries enrolled in 3 different health plans. The CMR completion rate for 3 models of patient outreach used for 3 different health plans over the service period was compared. The CMR completion rate for this study was calculated using the number of completed CMRs and the total number of beneficiaries who were contacted at least once for the provision of a CMR for each respective model by the MTMCCC. The CMR completion rate calculated for this study is modified slightly from the CMS definition because of limitations in patient population assignment to the MTMCCC.

RESULTS: The model in which patients were previously offered and subsequently completed a CMR from their health plans before contact by the MTMCCC had the highest CMR completion rate at $49.8 \%$, compared with $40.5 \%$ and $23.8 \%$ for the other 2 outreach models.

CONCLUSIONS: Offering patients a CMR, and subsequent completion, before provision of MTM services may increase CMR completion rate for health plans.

J Manag Care Spec Pharm. 2016;22(7):796-800

Copyright $\odot 2016$, Academy of Managed Care Pharmacy. All rights reserved.

\section{What is already known about this subject}

The comprehensive medication review (CMR) completion rate is currently a display measure for Medicare Part D health plans required by the Centers for Medicare \& Medicaid Services.

There are several approaches to patient care outreach for completing CMRs that are being used by health plans and medication therapy management (MTM) vendors.

\section{What this study adds}

This study revealed that use of a patient outreach model in which patients are offered, and subsequently complete, CMRs before the provision of MTM services may increase the CMR completion rate. Study findings provide guidance for design and implementation of patient outreach models that will help increase overall CMR completion rates.

$\mathrm{M}$ edication therapy management (MTM) programs were first introduced in 2003 with the implementation of the Medicare Prescription Drug, Improvement, and Modernization Act (MMA). ${ }^{1}$ At that time, there was no clear definition for how medication therapy management (MTM) services within MTM programs would be provided. Health plans had no specific guidelines to use in establishing their MTM programs; instead, the MMA allowed health plans to create their own enrollment criteria as long as they met the minimum requirements as set by the Centers for Medicare $\&$ Medicaid Services (CMS). ${ }^{1}$ Since 2003, requirements and facilitation of MTM programs have expanded and evolved. CMS has since established minimum requirements for patient population eligibility criteria based on number of Part D-covered medications, quantity of targeted chronic disease states, and expected annual Part D medication costs. ${ }^{1}$ For 2014, which is the year of this study period, the enrollment criteria were as follows 2 : (a) annual estimated minimum cost threshold for Part D medications of $\$ 3,017.00$; (b) at least 2-3 chronic disease states; and (c) at least 2-8 Part D-covered medications.

For the listed criteria that indicates a range for enrollment eligibility, health plans are able to determine the specific requirements based on their individual populations. For chronic disease states, health plans may choose to include any chronic disease state or may specify which chronic disease 
Evaluation of Comprehensive Medication Review Completion Rates Using 3 Patient Outreach Models

TABLE 1 Description of Patient Outreach Models and Populations

\begin{tabular}{c|l|l}
\hline Model & \multicolumn{1}{|c|}{ Patient Population } & \multicolumn{1}{c}{ Description } \\
\hline 1 & $\begin{array}{l}\text { Medicare Advantage } \\
\text { Prescription Drug Plan (MAPD) }\end{array}$ & $\begin{array}{l}\text { Beneficiaries received only an initial MTM program welcome letter from their health plans. Beneficiaries were } \\
\text { contacted via telephone by the MTMCCC at a later date to explain the MTM program and offer beneficiaries CMRs. }\end{array}$ \\
\hline 2 & $\begin{array}{l}\text { Medicare Prescription Drug Plan } \\
\text { (MAPD and PDP) }\end{array}$ & $\begin{array}{l}\text { Beneficiaries received an initial MTM program welcome letter from their health plans and/or a telephone } \\
\text { call from an outside call vendor to explain the MTM program and offer the CMR. If beneficiaries accepted } \\
\text { the CMR offer and were assigned to the MTMCCC, the MTMCCC provided this service by calling at a later } \\
\text { date to conduct the CMR, which was either completed at the time of the call or an appointment was made for } \\
\text { completing the CMR on a subsequent call. }\end{array}$ \\
\hline 3 & Medicaid & $\begin{array}{l}\text { Beneficiaries did not receive any information from their health plans regarding the MTM program. Beneficiaries } \\
\text { were contacted directly by the MTMCCC to explain the MTM program and offer a CMR. The CMR was } \\
\text { scheduled for a future date and time. }\end{array}$ \\
\hline
\end{tabular}

CMR= comprehensive medication review; MTM= medication therapy management; MTMCCC = University of Florida Medication Therapy Management Communication and Care Center.

states they will use for enrollment as long as they target at least 4 of the core disease states as listed by CMS. ${ }^{1}$ In 2014, 97.1\% of MTM programs targeted specific disease states, and the top 4 targeted disease states were diabetes, chronic heart failure, dyslipidemia, and hypertension. ${ }^{2}$ Similarly, plans may include any number of Part D medications as long as they meet the minimum range. ${ }^{1}$ Approximately $51.7 \%$ of 2014 programs targeted beneficiaries who filled at least 8 covered Part D medications, and $63.3 \%$ of programs required that those medications were for specified chronic conditions. ${ }^{2}$ MTM programs are also designed as opt-in enrollment, which means that if patients meets all of the eligibility criteria, they are automatically enrolled in an MTM program unless they specifically request not to be enrolled (opt-out). ${ }^{2}$ The flexibility of the MTM eligibility criteria allows for MTM programs to vary greatly from one health plan to another. Plans are also required to follow specific documentation instructions using CMS standardized templates for patient materials generated from MTM services.

CMS has also established program evaluation strategies for MTM programs. Health plans are currently evaluated on medication-related quality initiatives using a "star ratings" system. Star ratings are created from performance measures that contribute to the overall rating for individual health plans. ${ }^{3}$ The rating given is based on a scale of 1-5 stars, with 5 stars as the highest quality rating that health plans can achieve. In addition to star ratings, CMS also uses "display measures" as a means to evaluate health plan quality of services. ${ }^{3}$ Display measures are similar to the star performance measures in that they provide further quality evaluation of health plans; however, display measures are not included in the calculation of a plan's overall star rating. ${ }^{3}$ Star ratings and display measures are visible to consumers and may positively or negatively impact a health plan's ability to enroll patients, as well as the ability to obtain quality bonus payments. ${ }^{3}$ Display measures are often converted to star measures. The completion rate of comprehensive medication reviews (CMRs) was previously a display measure and is now a star performance measure as of 2016.,
Consequently, CMR completion rates has gained importance and attention from health plans, which are now motivated to find effective ways of positively impacting this particular performance measure.

CMS defines a CMR professional service as "an interactive person-to-person or telehealth medication review and consultation conducted in real-time between the patient and/or other authorized individual, such as prescriber or caregiver, and the pharmacist or other qualified provider; and is designed to improve patients' knowledge of their prescriptions, overthe-counter (OTC) medications, herbal therapies and dietary supplements, identify and address problems or concerns that patients may have, and empower patients to self-manage their medications and their health conditions." The CMR completion rate as defined by CMS is calculated by dividing the number of beneficiaries from the denominator who received a CMR during the reporting period by the total number of nonhospice beneficiaries who were at least aged 18 years or older at the beginning of the reporting period, met specified targeting criteria, and were enrolled in the MTM program for at least 60 days during the reporting period. ${ }^{4}$ The average CMR completion rate for Medicare Advantage Prescription Drug (MAPD) plans was $22.13 \%$ (range $=0 \%-88.50 \%$ ) for $2014 .{ }^{6}$

A CMR may be completed via numerous methods, including telephonic interviews, face-to-face encounters, or other telehealth technologies. Most health plans use mailings and/or telephone calls to patients that introduce the MTM program, as well as initiate the offering of CMR services. In 2014, 95.8\% of MTM programs offered CMR consultation telephonically. ${ }^{2}$ While some may hypothesize that the provision of services using telephonic means may increase the number of beneficiaries that can be contacted, there are challenges in reaching patients and completing the CMR depending on the employed outreach approach. At this time, there are minimal data available regarding which outreach approach is the most effective at achieving the highest possible CMR completion rate. 


\begin{tabular}{c|c|c|c}
\hline TABLE 2 & $\begin{array}{c}\text { MTM Eligibility Criteria for Each } \\
\text { Outreach Model }\end{array}$ \\
Model & $\begin{array}{c}\text { Annual Cost } \\
\text { Threshold }\end{array}$ & $\begin{array}{c}\text { Number of Chronic } \\
\text { Disease States }\end{array}$ & $\begin{array}{c}\text { Number of Part D } \\
\text { Medications }\end{array}$ \\
\hline 1 & $\$ 3,017.00$ & 3 & 7 \\
\hline 2 & $\$ 3,017.00$ & 3 & 7 \\
\hline 3 & None & None & Not applicable \\
\hline \multicolumn{4}{|c}{ MTM= medication therapy management. } \\
\hline
\end{tabular}

The University of Florida Medication Therapy Management Communication and Care Center (MTMCCC) is an MTM vendor that provides services for numerous managed care organizations telephonically, including the provision of CMRs. The purpose of this study was to compare the CMR completion rate, using a modified calculation for CMR completion rate, of 3 different patient outreach models used by the MTMCCC for beneficiaries enrolled in 3 different health plans.

\section{Methods}

\section{Design and Setting}

A retrospective chart analysis was conducted using data obtained from completed CMRs at the MTMCCC for health insurance beneficiaries enrolled in 3 health plans during the respective service periods for 2014. In this study, the following 3 models of patient outreach were compared (Table 1):

Model 1: A cold call was placed to patients who received an initial welcome letter from the health plan and was never previously offered a CMR before contact by the MTMCCC.

Model 2: Patients were initially offered, and subsequently completed, a CMR from the health plan before contact by the MTMCCC.

Model 3: Patients received no contact attempt from their health plans before receiving a call from the MTMCCC and were then scheduled to complete the CMR at a future date.

This analysis included all health plan beneficiaries who were eligible for CMRs, as determined by the MTM program enrollment criteria for each individual health plan, who were assigned to the MTMCCC, and who were subsequently contacted at least once for the provision of a CMR (Table 2). Each health plan supplied the MTMCCC with a list of eligible patients. Each patient was contacted telephonically at least once for the provision of a CMR. If patients could not be reached by phone and had a voice answering option available, a general message was left with a return phone number so patients could call the MTMCCC at their convenience. If the phone numbers were invalid, MTMCCC staff would attempt to obtain correct phone numbers by contacting patients' primary care physicians and/or preferred pharmacies. This study was approved by the University of Florida's Institutional Review Board.

\section{TABLE 3 CMR Completion Rate by Model}

\begin{tabular}{c|c|c|c}
\hline $\begin{array}{l}\text { Outreach } \\
\text { Model }\end{array}$ & $\begin{array}{c}\text { Number of } \\
\text { Completed CMRsa }\end{array}$ & $\begin{array}{c}\text { Total Number of } \\
\text { Patients Attempted }\end{array}$ & $\begin{array}{c}\text { CMR Completion } \\
\text { Rate }\end{array}$ \\
\hline 1 & 939 & 2,319 & $40.5 \%$ \\
\hline 2 & 6,327 & 12,710 & $49.8 \%$ \\
\hline 3 & 163 & 686 & $23.8 \%$ \\
\hline
\end{tabular}

${ }^{a}$ Numerical depiction of the different models of outreach with corresponding CMR completion rate percentages.

$C M R=$ comprehensive medication review

\section{Outcomes}

The primary outcome of this study was the CMR completion rate. The completion rate used for this study was modified slightly from the CMS criteria for the CMR completion rate calculation because of the population data available to MTMCCC.

\section{Calculation}

The CMS calculation for CMR completion rate includes all eligible patients enrolled in the MTM program for a health plan in the denominator. The CMR completion rate for this study was modified from the CMS definition and calculated using the number of CMRs completed by the MTMCCC divided by the total number of beneficiaries provided by the health plan to the MTMCCC who were eligible for the provision of a CMR for each respective model. The CMR completion rate calculated in this study is not a direct reflection of each health plan, since the MTMCCC was not assigned the entire population enrolled in the MTM program for each health plan. The total population eligible for accurately calculating the CMS-defined CMR completion rate was not available for analysis. Statistical significance between each model was analyzed using the chi-square test.

\section{Results}

\section{Primary Outcome}

For the 3 health plans examined, 7,429 CMRs were completed by the MTMCCC, with an attempt to reach 15,715 patients at least once for the provision of a CMR (Table 3) during the service period. Model 1 saw the second highest completion rate at $40.5 \%$. Model 2 demonstrated the highest acceptance rate at 49.8\%. Model 3 had the lowest CMR acceptance rate at 23.8\% (Figure 1). The chi square test demonstrated that the models were statistically significantly different from one another, as shown in Table 4.

\section{Discussion}

This study compared the CMR completion rate for 3 models of patient outreach used for 3 health plans at the MTMCCC. Based on data analysis, the CMR completion rate for the 3 outreach models ranged from $27.8 \%$ to $49.8 \%$ using the modified calculation. The average CMR completion rate as reported by CMS was $22.13 \%$ for MAPD plans in $2014 .{ }^{6}$ The CMR 


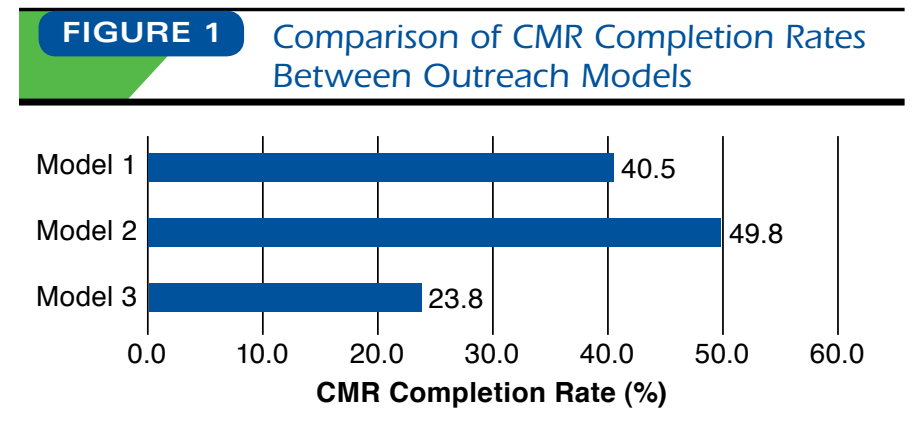

$C M R=$ comprehensive medication review.

completion rate is now a performance measure as of 2016, which will affect the overall star ratings of health plans. Past performance and analysis of CMR completion rate gathered as a display measure have been used by CMS to establish the levels of performance that distinguish the rating levels from 1 to 5 stars. ${ }^{7}$ A CMR completion rate for a health plan that exceeds the CMS average rate when compared with other plans may predict an above average star rating for this measure. This study demonstrated that all 3 of the outreach models used by the MTMCCC were effective in accomplishing a CMR completion rate of at least $27.8 \%$; however, it should be noted that by using a modified calculation for CMR completion rate (as was used in this study), the modified calculation may cause an overly inflated CMR completion rate when compared with the CMS CMR completion rate calculation.

The model with the highest completion rate was Model 2. Patients in Model 2 were initially offered, and subsequently completed, CMRs offered by their health plans before contact by the MTMCCC. The higher performance rate for this outreach model in comparison with the other models is likely explained by a patient's familiarity, and potential expectation, of a CMR. This previous knowledge of MTM services could have increased patient willingness to answer the phone and subsequently participate in the CMR as provided by the MTMCCC. In comparison, patients in Model 1 only received an initial welcome letter and were never offered MTM services before contact by the MTMCCC. Similarly, patients in Model 3 had no previous information about MTM services before initial contact by the MTMCCC. These patients were likely unfamiliar with and possibly not expecting the outreach attempt from the MTMCCC; therefore, they were less likely to accept and complete a CMR.

With the addition of CMR completion rates to the star rating system, the overall ratings of health plans, as well as potential bonus payments, will be directly affected. Effective use of resources and staffing to engage patients enrolled in MTM programs will be of increasing interest and importance to health plans. The results of this study suggest that the offering of CMRs to patients, with subsequent completion, before the provision of MTM services may increase the CMR completion rate when compared with other models of patient outreach. This study also demonstrated a statistically significant

\section{TABLE 4 Chi-Square Statistical Test for} Significance

\begin{tabular}{l|c|c}
\hline Comparison & Chi-Square Statistic & $P$ Value \\
\hline All 3 groups & 226.9716 & $<0.00001$ \\
\hline Group 1 vs. 2 & 67.7495 & $<0.00001$ \\
\hline Group 2 vs. 3 & 176.4197 & $<0.00001$ \\
\hline Group 1 vs. 3 & 63.8080 & $<0.00001$ \\
\hline
\end{tabular}

benefit to reaching patients directly with the offering of a CMR compared with only receiving a welcome letter and no direct patient communication regarding the CMR. It is likely that the use of a multimodal approach, such as mailings and telephone contact, may increase the CMR completion rate.

\section{Limitations}

While conducting this research, a number of limitations were identified. The first limitation was the calculation used for the CMR completion rate. The modified calculation used only takes into account the patient population that was assigned to the MTMCCC, not the entire MTM-eligible population of the health plans. In one of the models used, only a portion of the patients who had been offered MTM services and subsequently agreed to receiving MTM services were allocated to the MTMCCC. In another model, the MTMCCC was used as a back-up MTM vendor; however, it did receive the entire MTM-eligible population for the provision of MTM services. For this particular health plan, there were a few instances of patients who had already completed a CMR with the health plan's main MTM vendor, so services were not provided again. The lack of services provided to these patients affected the modified CMR completion rate.

Another limitation was the variance among the patient populations for each health care plan included in this study. Patients in these 3 health plans differed based on the health plan enrollment and MTM eligibility criteria. For example, a greater number of patients in Model 1 are still in the workforce compared with the other populations. Patients who are employed may be limited in the amount of time they have available to interact with a pharmacist for the completion of a CMR. Those patients who work outside the home may also be harder to reach by telephone because of the limited amount of time at home during which the MTMCCC provides services, compared with patients in other models who do not work outside the home. Patients in Model 3 had the least financial income among the 3 models. Also, some of the patients in Model 3 had government-provided cell phones or had limited minutes on their phones, so they were less likely to engage in a telephone conversation about their medications. Additionally, of the 3 populations, there was a greater number of foreign language speakers in Model 2 , which could have decreased the success rate of completing CMRs because of language barriers or patient unwillingness to use an interpreter or translator. These differences could be potential confounders in a patient's ability, or willingness, to be contacted by or participate in the CMR service. 
An additional limitation was the number of patient contact attempts made for each model. In Model 2, patients were contacted at least 3 times for the completion of a CMR. In contrast, patients in Models 1 and 3 may have only received 1 contact attempt during the service period. The lack of contact attempts in Models 1 and 3 were a result of the limited time frame for service provision, as well as contracting language with the different health plans, which limited the number of contact attempts per patient. Consequently, patients in Model 2 had more opportunities to potentially accept the services when compared with the other 2 models.

Another potential limitation was the challenge of continuity of care for patients with the provision of MTM services from the MTMCCC. Patients enrolled in their health plans' MTM programs who remained eligible for MTM services continued to receive CMR offerings from the MTMCCC on a yearly basis. Patients enrolled in health plans for Models 2 and 3 may have completed CMRs with the MTMCCC pharmacist during a previous year. These previous encounters may have increased the likelihood of these patients answering phone calls and completing CMRs with the MTMCCC.

A final limitation was the potential for patients to avoid an outreach attempt because of medication adherence issues. Patients in Model 1 were targeted for MTM services based on known medication adherence concerns. These patients were identified with adherence issues as determined by a low proportion of days covered for their medications and were also contacted for MTM services. Contact attempts by the MTMCCC were displayed to the patients as the names of the health plans and their telephone numbers through caller identification services. These patients may have chosen not to accept calls from the MTMCCC, on behalf of their health plans, for MTM services because of nonadherence to their medication schedules.

\section{Conclusions}

This study set out to determine the differences in success between 3 models of outreach in the completion of CMRs during the provision of MTM services. Patients who were offered, and subsequently completed, CMRs before contact for provision of MTM services had a higher CMR acceptance rate when compared with the models where the patient population had less, or no, introduction to MTM programs. The higher rate of acceptance by patients in Model 2 is attributed to the patients' familiarity with the service and the expectation of being offered CMRs. This study supports introducing and offering CMR services before patients are contacted for the provision of MTM services. It also appears that a multimodal outreach attempt, including mailings and several phone calls, may result in a higher overall CMR completion rate for health plans.

Future directions recommended for this topic include the comparison of the employed patient outreach models to a similar group of health plan beneficiaries. Additionally, a future study could examine the best method for initially offering a CMR (i.e., written or verbal) by the health plan in order to increase CMR acceptance rates when the service is provided.

\section{Authors}

DARYL E. MILLER, PharmD; TERESA E. ROANE, PharmD, BCACP; JENNIFER A. SALO, PharmD, BCACP; and HEATHER C. HARDIN, PharmD, BCACP, Medication Therapy Management Communication and Care Center, University of Florida College of Pharmacy, Gainesville.

AUTHOR CORRESPONDENCE: Teresa E. Roane, PharmD, BCACP, Medication Therapy Management Communication and Care Center, University of Florida College of Pharmacy, 2046 N.E. Waldo Rd., Office 3123, Gainesville, FL 32609. Tel.: 352.273.9692;

E-mail:troane@cop.ufl.edu.

\section{DISCLOSURES}

No outside funding supported this research. The University of Florida (UF) Medication Therapy Management Communication and Care Center (MTMCCC) receives grant funding from the Agency for Health Care Administration (AHCA) for services. Roane and Hardin are currently employed by UF as faculty with their practice sites at the UF MTMCCC. Salo is currently employed at UF as a clinical pharmacist with her practice site at the UF MTMCCC. Miller is currently employed as a transition of care clinical pharmacist at St. Joseph's Hospital in Tampa, Florida. The authors declare no potential conflicts of interest with respect to the research, authorship, and/or publication of this article.

Miller and Roane were primary contributors to study concept and design along with Salo and Hardin. Miller and Roane took the lead in data collection, assisted by Salo and Hardin, and data interpretation was performed by all the authors. The manuscript was written by all the authors and revised primarily by Roane and Miller, along with Salo and Hardin.

\section{REFERENCES}

1. Medicare Prescription Drug, Improvement, and Modernization Act of 2003. Public Law 108-173. December 8, 2003. Available at: http://www.gpo.gov/fdsys/ pkg/PLAW-108publ173/pdf/PLAW-108publ173.pdf. Accessed May 11, 2016.

2. Centers for Medicare \& Medicaid Services. 2014 Medicare Part D medication therapy management (MTM) programs. Fact sheet: summary of 2014 MTM programs. August 21, 2014. Available at: http://www.cms. gov/Medicare/Prescription-Drug-Coverage/PrescriptionDrugCovContra/ Downloads/CY2014-MTM-Fact-Sheet.pdf. Accessed May 11, 2016.

3. Tudor $\mathrm{C}$. Memo. Request for comments regarding enhancements to the Part C and Part D star ratings. Department of Health \& Human Services, Centers for Medicare \& Medicaid Services. November 30, 2012. Available at: http://www.cms.gov/Medicare/ Prescription-Drug-Coverage/PrescriptionDrugCovContra/Downloads/ CommentsforPartCandDStarRatings.pdf. Accessed May 11, 2016.

4. Centers for Medicare \& Medicaid Services. Medicare 2016 Part $C$ and D star rating technical notes. First plan preview. Draft. August 5, 2015. Available at: https://www.cms.gov/Medicare/Prescription-Drug-Coverage/ PrescriptionDrugCovGenIn/Downloads/2016-Technical-Notes-Preview1-v2015_08_05.pdf. Accessed May 11, 2016.

5. Shapiro J. Memo. CY 2015 Medication therapy management program guidance and submission instructions. Department of Health and Human Services, Centers for Medicare \& Medicaid Services. May 7, 2014.

Available at: http://www.cms.gov/Medicare/Prescription-Drug-Coverage/

PrescriptionDrugCovContra/Downloads/MemoContractYear2015

MedicationTherapyManagementProgramSubmission050714.pdf. Accessed May 11, 2016.

6. Centers for Medicare \& Medicaid Services. 2015 Part C \& D display measures_v020615. Part C and D performance data. Available at: http://www. cms.gov/Medicare/Prescription-Drug-Coverage/PrescriptionDrugCovGenIn/ PerformanceData.html. Accessed May 11, 2016

7. Centers for Medicare \& Medicaid Services. Trends in Part C \& D star rating measure cut points. November 18, 2014. Available at: http://www.cms. gov/Medicare/Prescription-Drug-Coverage/PrescriptionDrugCovGenIn/ Downloads/2015-Trends-in-Part-C-and-D-Star-Rating-Measure-Cut-Points-. pdf. Accessed May 11, 2016. 